\title{
Referral for intestinal transplantation: four-year tertiary centre experience
}

\author{
P. Paskaran, A. U. Murugananthan, D. A. J. Lloyd, J. M. D. Nightingale, S. J. Middleton \\ and S. M. Gabe \\ Lennard-Jones Intestinal Failure Unit, St Mark's Hospital, Harrow, UK
}

\begin{abstract}
Current Intestinal Transplantation (ITx) criteria do not appear to select adult patients on Home Parenteral Nutrition (HPN) with a poor prognosis. Mortality rates over a 3-year period are comparable in those meeting criteria for ITx and those that do not ${ }^{(1)}$. We present 4 -year data on (i) the number of patients receiving HPN at St Mark's Hospital meeting criteria for ITx, (ii) patients referred for ITx and (iii) mortality rates.

Patients receiving HPN in 2005 were identified and then reviewed annually for 4 years for eligibility for referral for ITx based on fulfilment of $\geq 1$ of the following approved criteria ${ }^{(2)}$.

- advanced PN-associated liver disease (PNALD)

- thrombosis of $\geq 2$ major veins

- frequent CVC sepsis ( $\geq 2$ episodes in the previous 12 months, fungaemia or septic shock)

- frequent severe dehydration

- patient request/quality of life
\end{abstract}

Our cohort consisted of 123 patients receiving HPN in the first year falling in subsequent years to 122, 117 and 103 . Twelve patients died over the 4-year period, 3 meeting criteria and 9 not meeting ITx criteria. Despite the fall in patient numbers from either death or discontinuation of HPN, referral rates remained static or reduced with $19 \%$ of patients referred in year one followed by $23 \%, 25 \%$ and $22 \%$ in the subsequent years. We refer all patients with PNALD ITx criteria, approximately $25 \%$ of those with thrombosis criteria and $2 \%$ of those with CVC sepsis criteria. There was no significant difference in annual death rate between patients meeting and not meeting ITx criteria.

Our referral rate for ITX remains significantly lower than the number of patients meeting criteria for ITX. Crucially, we show no survival difference between those meeting and not meeting ITx criteria at 4 years suggesting that current ITx guidelines do not appear to select adult patients with high mortality.

1. Paskaran P, Murugananthan AU, Ralphs SJ et al. (2008) Clinl Nutr 3(Suppl. 1), 158. 